\title{
Bubble-Nucleation Rates for Cosmological Phase Transitions
}

\author{
Alessandro Strumia \\ Dipartimento di Fisica, Università di Pisa \\ e INFN, sezione di Pisa, I-56126 Pisa, Italia \\ and \\ Nikolaos Tetradis \\ Scuola Normale Superiore \\ Piazza dei Cavalieri 7, I-56126 Pisa, Italia
}

\begin{abstract}
We estimate bubble-nucleation rates for cosmological phase transitions. We concentrate on the evaluation of the pre-exponential factor, for which we give approximate analytical expressions. Our approach relies on the use of a real coarse-grained potential. We show how the coarse-graining scale can be determined in the studies of high-temperature phase transitions. We discuss the metastability bound on the Higgs-boson mass and the electroweak phase transition. We find that the saddlepoint approximation is reliable in the first case and breaks down in the second case.
\end{abstract}

Introduction: The estimates of bubble-nucleation rates for cosmological first-order phase transitions are carried out within Langer's theory of homogeneous nucleation $\mathbb{1}$, applied to relativistic field theory in refs. [2]. The nucleation rate is exponentially suppressed by the action (free energy rescaled by the temperature) of the critical bubble, a saddle point of the free energy of the system. Significant contributions to the nucleation rate may arise from higher orders in a systematic expansion around this saddle point. The first correction has the form of a pre-exponential factor that involves fluctuation determinants around the saddle-point configuration and the false vacuum. The evaluation of this factor is a difficult problem at the conceptual and technical level, as crucial issues associated with the convexity of the potential, the divergences of the fluctuation determinants and the double-counting of the effect of fluctuations must be resolved. Several approaches have been proposed in order to address these issues [3, 4].

In a series of recent works [5]- 8 , following the proposal of refs. [9], we developed a consistent approach, based on the effective average action $\Gamma_{k}$ [10 that can be interpreted as a coarse-grained free energy. Fluctua- tions with characteristic momenta larger than a coarsegraining scale $\left(q^{2} \gtrsim k^{2}\right)$ are integrated out and their effect is incorporated in $\Gamma_{k}$. In the limit $k \rightarrow 0, \Gamma_{k}$ becomes equal to the effective action. The $k$ dependence of $\Gamma_{k}$ is described by an exact flow equation 11]. This flow equation can be translated into evolution equations for functions appearing in a derivative expansion of the action [12]. An approximation that is sufficient in most cases takes into account the effective average potential $U_{k}$ and a standard kinetic term and neglects higher derivative terms in the action. The bare theory is defined at some high scale $\Lambda$ that can be identified with the ultraviolet cutoff. It is, however, more convenient to choose a starting scale $k_{0}$ below the temperature $T$, where the effective average action of a $(3+1)$-dimensional theory at non-zero temperature can be described in terms of an effective three-dimensional action at zero temperature $13,14$.

In ref. 55 we computed the form of $U_{k}$ at scales $k \leq k_{0}$ for a theory of one scalar field by integrating its evolution equation, starting with an initial potential $U_{k_{0}}$ with two minima separated by a barrier. $U_{k}$ is real and non-convex for non-zero $k$, and approaches convex- 
ity only in the limit $k \rightarrow 0$. The nucleation rate must be computed for $k$ larger than the scale $k_{f}$ for which the negative curvature at the top of the barrier becomes approximately equal to $-k_{f}^{2}$ 15, 16]. For $k<k_{f}$ the form of the potential is affected by field configurations that interpolate between the two minima. Also, for $k \gtrsim k_{f}$ the typical length scale of a thick-wall critical bubble is $z 1 / k$. We performed the calculation of the nucleation rate for a range of scales above and near $k_{f}$. In our approach the pre-exponential factor is calculated with an ultraviolet cutoff of order $k$, as the effect of fluctuations with $q^{2} \gtrsim k^{2}$ is already incorporated in $U_{k}$. The saddle-point configuration has an action $S_{k}$ with a significant $k$ dependence. For strongly first-order phase transitions, the nucleation rate $I=A_{k} \exp \left(-S_{k}\right)$ is dominated by the exponential suppression. The main role of the prefactor $A_{k}$, which is also $k$ dependent, is to remove the scale dependence from the total nucleation rate. For progressively more weakly first-order phase transitions, the difference between $S_{k}$ and $\ln \left(A_{k} / k_{f}^{4}\right)$ diminishes and contributions from higher orders in the expansion around the saddle point become important. At the same time, a significant $k$ dependence of the predicted nucleation rate develops. The prefactor always enhances the total nucleation rate and can compensate the exponential suppression for weakly first-order phase transitions. This indicates that there is a limit to the validity of Langer's picture of homogeneous nucleation, set by the requirement of convergence of the expansion around the saddle-point [6].

First-order phase transitions in two-scalar models were studied in ref. [7], where the applicability of homogeneous nucleation theory to radiatively-induced firstorder phase transitions was tested. The prefactor tends to suppress the total nucleation rate in this case. It was found that the expansion around the saddle point is not convergent for such phase transitions, for which fluctuations are so strong that they generate a new minimum in the potential. This indicates that estimates of bubble-nucleation rates for the electroweak phase transition that are based only on the saddle-point action may be very misleading. The reliability of our approach was reconfirmed through the study of $(2+1)$ dimensional theories at non-zero temperature 8 and the comparison with results from lattice simulations.

In this paper we present a simplified description of our approach that permits quick tests of the applicability of nucleation theory in a variety of problems. We give approximate expressions for the evaluation of the pre-exponential factor in the nucleation rate:

- Eq. (3) allows a quick determination of the coarsegrained potential in terms of the standard perturbative potential.

- Eq. (6) allows an approximate evaluation of the prefactor in terms of the critical-bubble profile.
We check their validity by comparing them with numerical calculations of the fluctuation determinants. Finally, we estimate the importance of the prefactor in the cases of the bound on the Higgs-boson mass from vacuum stability and the electroweak phase transition.

Simple approximation for the coarse-grained potential: We study the potential of a scalar field $\phi$ coupled to several other fields with zero expectation value. We consider the theory at energy scales below the temperature $T$, so that a $(3+1)$-dimensional theory at non-zero temperature can be described in terms of an effective three-dimensional one at zero temperature. The correspondence between the quantities we use and the ones of the four-dimensional theory is given by

$$
\phi=\frac{\phi_{4}}{\sqrt{T}}, \quad U(\phi)=\frac{U_{4}\left(\phi_{4}, T\right)}{T} .
$$

The evolution equation for the coarse-grained potential $U_{k}(\phi)$ takes the form [1], 12, 6, 6, []

$$
\frac{\partial}{\partial k^{2}} U_{k}(\phi)=-\frac{1}{8 \pi} \operatorname{Tr} \sqrt{k^{2}+\mathcal{M}_{k}^{2}(\phi)},
$$

where $\mathcal{M}_{k}^{2}(\phi)$ is the mass matrix of all the fields whose mass depends on $k$ and the expectation value of $\phi$. In order to derive the above expression we assumed that the various fields have standard kinetic terms and neglected higher-derivative interactions in the effective average action. We also used a mass-like infrared cutoff $\sim k^{2}$ in order to eliminate contributions to $U_{k}$ from modes with characteristic momenta $q^{2} \lesssim k^{2}$.

The first step of an iterative solution of eq. (2) gives 17]

$$
\begin{gathered}
U_{k}^{(1)}(\phi)=U_{k_{0}}(\phi)+\frac{1}{12 \pi} \times \\
\times \operatorname{Tr}\left\{\left[k_{0}^{2}+\mathcal{M}_{k_{1}}^{2}(\phi)\right]^{\frac{3}{2}}-\left[k^{2}+\mathcal{M}_{k_{1}}^{2}(\phi)\right]^{\frac{3}{2}}\right\} .
\end{gathered}
$$

The scale $k_{1}$ can be chosen arbitrarily between $k_{0}$ and $k$. For $k=0$ and $k_{1}=k_{0}$, eq. (3) is a regularized one-loop approximation to the effective potential. For $k_{1}=k>0$, it takes the form of a "mass-gap" equation for the coarse-grained potential. It is the latter form that we shall find more useful.

For practical applications of our method the values of the scales $k_{0}$ and $k_{f}$ must be specified. For studies of high-temperature field theories the natural choice of $k_{0}$ is $k_{0}=T$. For $k \lesssim k_{0}$ the system can be described in terms of an effective three-dimensional theory at zero temperature [13, 14]. The form of $U_{k_{0}}(\phi)$ depends on the zero-temperature potential and the thermal fluctuations with $q^{2}>T^{2}$. It can be determined by integrating the evolution equations for the $(3+1)$-dimensional theory at zero and non-zero temperature, starting from a scale $\Lambda \gg k_{0}$, with the bare action as initial condition [13]. In refs. 13, 14] it was checked that eq. (3) 
with $k=0$ and $k_{1}=k_{0}$ reproduces the one-loop approximation to the high-temperature effective potential. This fact suggests a simpler procedure that was employed with success in ref. [8]. One can demand that $U_{k}^{(1)}(\phi)$ is equal, at $k=0$, to the perturbative result (or the solution to a "mass-gap" equation). It is easy then to extract $U_{k_{0}}(\phi)$ from eq. (3). In this way no explicit reference to the zero-temperature potential and the thermal modes with $q^{2}>T^{2}$ is necessary. Notice that the imaginary part of the standard perturbative potential matches with the one of our approximate solution $U_{0}^{(1)}(\phi)$ of eq. (3).

The choice of $k_{f}$ is dictated by the form of the matrix $\mathcal{M}_{k_{1}}^{2}(\phi)$ in eq. (3). The eigenvalue $\mathcal{M}_{1 k_{1}}^{2}(\phi)$ that corresponds to the field that varies along the profile of the bubble is equal to the second derivative of the potential. This means that $U_{k}^{(1)}(\phi)$ would become complex in the non-convex regions for $k<k_{f}$, with $k_{f}^{2}=\max \left|U_{k_{1}}^{\prime \prime}{ }^{(1)}(\phi)\right|$. This reproduces the well-known pathology of perturbation theory. However, the exact solution $U_{k}(\phi)$ of eq. (2) does not have imaginary parts. On the contrary, after $k$ becomes almost equal (within a few percent) to $\max \left|U_{k}^{\prime \prime}(\phi)\right|$ in the non-convex regions, the negative curvature of the potential goes to zero $\sim-k^{2}$ 16, 27, resulting in the Maxwell construction. This behaviour is related to the renormalization of the potential by configurations in the path integral that interpolate between its minima [15]. As this mechanism is intrinsically non-perturbative, it is not captured by the approximate solution of eq. (3). As was demonstrated in refs. [5]- 9], a consistent description of tunnelling can be obtained by computing the nucleation rate at a coarse-graining scale sufficiently large, so that the potential is not affected by interpolating configurations and the "approach to convexity" has not set in. For our approximate discussion, the solution of eq. (3) can be employed and the nucleation rate can be computed above and close to $k_{f}$, with $k_{f}^{2}=\max \left|U_{k_{1}}^{\prime \prime}{ }^{(1)}(\phi)\right|$. If the expansion around the saddle point is convergent, the value of the bubblenucleation rate has a weak dependence on $k$.

Simple approximation for the nucleation rate: The nucleation rate is given by

$$
I=A_{k} \exp \left(-S_{k}\right)
$$

where

$$
\begin{aligned}
A_{k}= & \frac{E_{0}}{2 \pi}\left(\frac{S_{k}}{2 \pi}\right)^{3 / 2} \mid \frac{\operatorname{Det}^{\prime}\left[-\partial^{2}+\mathcal{M}_{k}^{2}\left(\phi_{\mathrm{b}}(r)\right)\right]}{\operatorname{Det}\left[-\partial^{2}+k^{2}+\mathcal{M}_{k}^{2}\left(\phi_{\mathrm{b}}(r)\right)\right]} \times \\
& \times\left.\frac{\operatorname{Det}\left[-\partial^{2}+k^{2}+\mathcal{M}_{k}^{2}(0)\right]}{\operatorname{Det}\left[-\partial^{2}+\mathcal{M}_{k}^{2}(0)\right]}\right|^{-1 / 2}
\end{aligned}
$$

Here $\phi_{\mathrm{b}}(r)$ is the profile of the spherically-symmetric saddle point, $S_{k}$ its action computed through the po- tential $U_{k}(\phi)$, and $\phi=0$ corresponds to the false vacuum. The prime in the fluctuation determinant around the saddle point denotes that the 3 zero eigenvalues of the operator $-\partial^{2}+\mathcal{M}_{k}^{2}\left(\phi_{\mathrm{b}}(r)\right)$, corresponding to displacements of the critical bubble, have been removed. $E_{0}$ is the square root of the absolute value of the unique negative eigenvalue. For theories with continuous internal symmetries, the corresponding zero eigenvalues are removed from the determinants as well and the nucleation rate is multiplied by an appropriate factor 18. The above form of $A_{k}$ guarantees that only modes with characteristic momenta $q^{2} \lesssim k^{2}$ contribute to the nucleation rate. We emphasize that the use of an ultraviolet cutoff in eq. (4) that matches the infrared cutoff procedure in the derivation of eq. (2) is crucial for the consistency of our approach. In both cases, mass-like cutoffs have been used.

The prefactor $A_{k}$ can be expressed as a product of terms $A_{i k}$ corresponding to eigenvalues $\mathcal{M}_{i k}^{2}$ of the mass matrix in orthogonal field directions. Also, using the spherical symmetry of the saddle-point configuration, one can express each of $A_{i k}$ as a product of contributions $c_{i \ell}$ with given quantum number $\ell$ [5]. For large $\ell$, first-order perturbation theory in $W_{i k}(r) \equiv$ $\mathcal{M}_{i k}^{2}\left(\phi_{b}(r)\right)-\mathcal{M}_{i k}^{2}(0)$ gives [5]

$$
c_{i \ell} \rightarrow 1+\frac{D_{i}}{\ell^{2}}+\mathcal{O}\left(\frac{1}{\ell^{4}}\right)
$$

with

$$
D_{i}=-\frac{1}{4} k^{2} \int_{0}^{\infty} r^{3} W_{i k}(r) d r .
$$

The above expressions usually gives a reasonable approximation of $c_{i \ell}$ for small values of $\ell$ as well. If this is the case, we can evaluate the infinite product and derive an approximate expression for the prefactors

$$
\ln A_{i k} \approx \operatorname{sign}\left(D_{i}\right) \sqrt{\left|D_{i}\right|} \pi
$$

We assume that $A_{i k}$ are measured in units of some dimensionful quantity of the order of the scale $k_{f}$. The precise determination of these units is not necessary because of the approximate nature of the above expression.

The validity of eq. (6) can be checked through a comparison with the numerical evaluation of the prefactors in refs. [5]- [7]. In fig. 1 we present four examples from these studies, in which we have added the predictions of eq. (6). Figs. $1 \mathrm{a}$ and $1 \mathrm{~b}$ correspond to the one-scalar theory of refs. [5, 6], for the same choice of parameters as in fig. 1 of ref. 6]. The initial potential at the scale $k_{0}$ has already two minima separated by a barrier. The form of the potential at lower scales is determined through the numerical integration of eq. (2), with $\mathcal{M}_{k}^{2}(\phi)=U_{k}^{\prime \prime}(\phi)$. The negative of the action of the saddle point $-S_{k}$ is denoted by diamonds, the prefactor $\ln \left(A_{k} / k_{f}^{4}\right)$ by stars and the nucleation rate $\ln \left(I / k_{f}^{4}\right)$ 

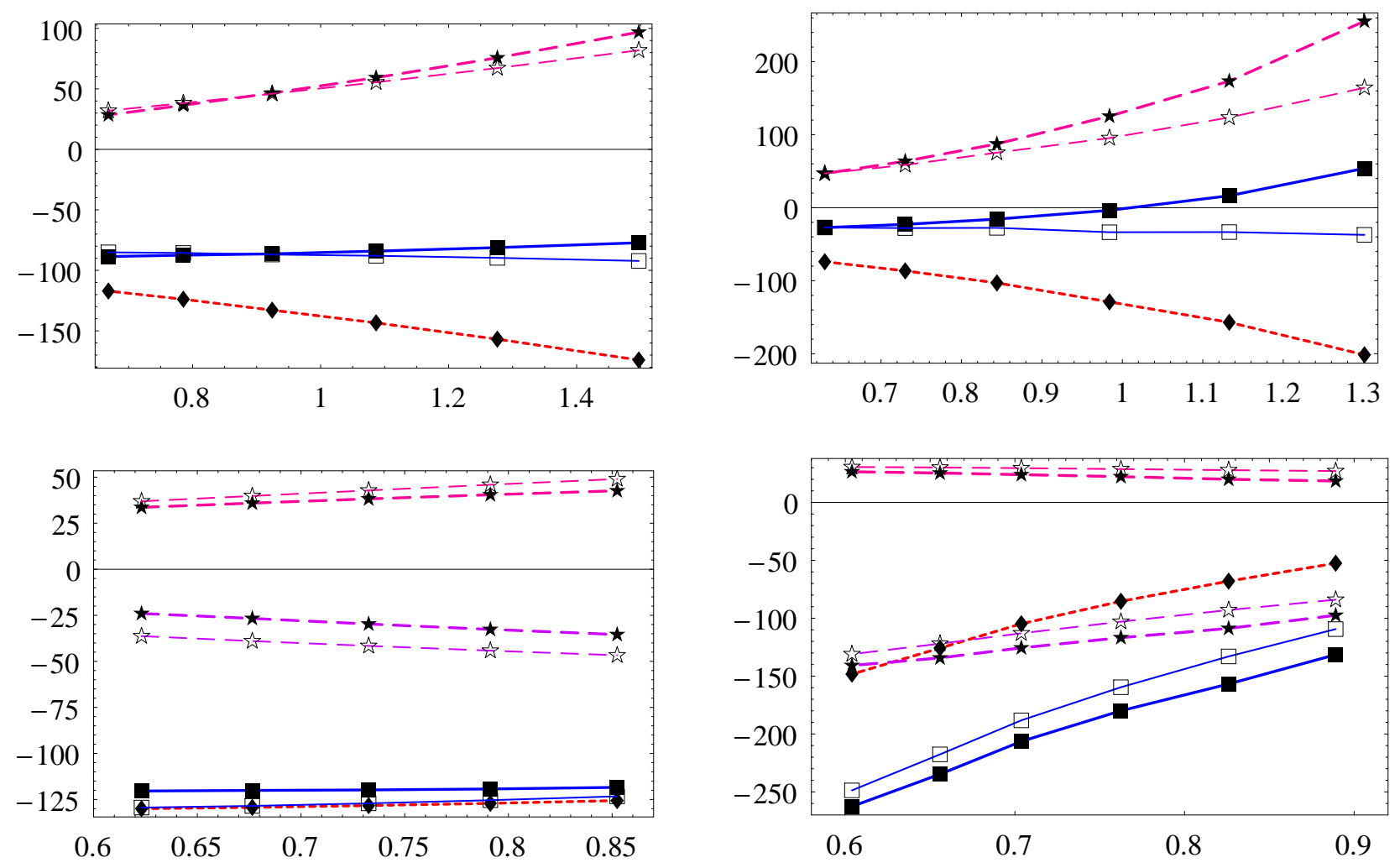

Figure 1: The negative of the action of the saddle point $-S_{k}$ (diamonds), the prefactor $\ln \left(A_{k} / k_{f}^{4}\right)$ (stars) and the nucleation rate $\ln \left(I / k_{f}^{4}\right)$ (squares), as a function of the scale $k$ in units of the field mass at the absolute minimum. The dark stars and squares denote values computed numerically, while the open stars and squares denote values obtained through the estimate of eq. (6). The first row corresponds to a theory of one scalar field, and the second row to a two-field theory.

by squares. The dark stars and squares denote values computed numerically, while the open stars and squares denote values obtained through the estimate of eq. (6). The above quantities are plotted as a function of the ratio $k / \sqrt{U_{k}^{\prime \prime}\left(\phi_{t}\right)}$, i.e. the scale $k$ in units of the field mass at the absolute minimum located at $\phi_{t}$. In the first case the expansion around the saddle point is convergent, while in the second one it breaks down. For both cases, we observe a good agreement between the exact and the approximate values of $\ln \left(A_{k} / k_{f}^{4}\right)$ for the range of scales near $k_{f}$.

In figs. 1c and 1d we present similar results for the two-scalar theory of ref. [7]. There are two prefactors, corresponding to the field $\phi_{1}$ that varies along the profile of the bubble, and the orthogonal field $\phi_{2}$ with zero expectation value. In the case of fig. 1c (corresponding to fig. 2 of ref. [7]), the potential at the scale $k_{0}$ has already two minima. In the case of fig. 1d (corresponding to fig. 4 of ref. [7]), the initial potential is $U_{k_{0}}=m_{k_{0}}^{2}\left(\phi_{1}^{2}+\phi_{2}^{2}\right) / 2+\lambda_{k_{0}}\left(\phi_{1}^{4}+\phi_{2}^{4}\right) / 4+g_{k_{0}} \phi_{1}^{2} \phi_{2}^{2} / 4$ with $g_{k_{0}} / \lambda_{k_{0}}=40$. The minimum at the origin appears at scales below $k_{0}$ and the first-order phase transition is radiatively induced [19]. In the first case the expansion around the saddle point is convergent, while in the second one, which is typical of all radiativelyinduced phase transitions we have studied, it breaks down. For both cases, we observe again a good agreement between the exact and the approximate values of the prefactor.

Metastability bound on the Higgs-boson mass: As a first example we apply our formalism to the question of the bound on the Higgs-boson mass from the stability of our vacuum 20-23]. The top-quark radiative corrections to the effective potential of the Higgs field may result in the appearance of a new minimum, deeper than the one located at $247 \mathrm{GeV}$. In order for this not to happen, a lower bound on the Higgs-boson mass must be imposed [20]. This bound can be relaxed

1 There is a difference of a factor of 2 in the definition of $\lambda$ between this work and ref. [7. 
if one allows for the presence of a new vacuum, but demands that the time needed for a transition to it is larger than the age of our universe. The largest rates are associated with thermal transitions at high temperatures?, from a metastable minimum in the symmetric phase of the Standard Model directly to the absolute minimum at very large Higgs-field expectation values $21-23$.

In our simplified discussion we neglect the contributions from the gauge fields to the effective potential of the Higgs field. The main effect of interest is associated with the top quark, while our estimates of the transition rate are approximate. As a result, a detailed discussion of the gauge-field contributions does not improve the accuracy of our conclusions. We can start by considering the effective three-dimensional description of the top-Higgs system at a scale $k_{0}=T$. At this scale the top-quark fluctuations are expected to be almost decoupled, as the absence of a zero Matsubara frequency for fermions implies. This permits a very simple determination of the potential $U_{k_{0}}(\phi)$, which is equal to the sum of the zero-temperature potential and the contribution from the top-quark thermal fluctuations. The latter can be approximated by the standard one-loop expression. There is also the contribution of the Higgs-boson thermal fluctuations with characteristic momenta $q^{2}>k_{0}^{2}[13$, 14], but their effect is small for the Higgs-boson masses of interest.

Following ref. 22, we keep only the leading temperature correction in the expansion of the one-loop thermal contribution from the top quark. By performing the rescaling of eqs. (1), we express the potential as

$$
U_{k_{0}}(\phi)=\frac{m^{2}}{2} \phi^{2}-\frac{\kappa}{4} \phi^{4}
$$

where $m^{2}=g_{t 4}^{2} T^{2} / 4, \kappa=\left|\lambda_{4}\right| T$, and $\lambda_{4}$ is the average (negative) value of the quartic coupling of the Higgs field over the region of Higgs-field expectation

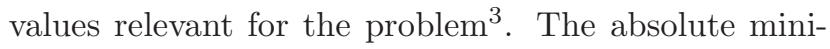
mum of the potential lies at Higgs-field values larger than the range over which the above approximation is valid. However, the profile of a critical bubble and the nucleation rate can be determined, exactly as in ref. 22. For the determination of the prefactor we need to specify the coarse-graining scale $k_{f}$. As the potential at the scale $k_{0}=T$ already has a metastable

2 The typical temperature at which the nucleation rate is maximized is of the order of the Higgs-field value at the absolute minimum. This can be larger than $247 \mathrm{GeV}$ by several orders of magnitude. It is implicitly assumed that such temperatures were realized in the early Universe, for example through efficient reheating after inflation. If this is not the case, the bound on the Higgs-boson mass is less stringent than the one we consider.

3 As the zero-temperature quartic coupling $\lambda_{4}$ varies only logarithmically with $\phi$, it can be approximated as constant over the range of field values that are relevant for the profile of the critical bubble 22 . minimum and a barrier, the choice $k_{f}=k_{0}$ seems justified.

Through the rescalings $r=\tilde{r} / m, \phi=\tilde{\phi} m / \sqrt{\kappa}$, the potential can be written as $\tilde{V}(\tilde{\phi})=\tilde{\phi}^{2} / 2-\tilde{\phi}^{4} / 4$. As a result, we need to determine the saddle-point profile numerically only once. The action of the saddle point can be written as 22

$$
S_{k_{0}}=\tilde{S} \frac{m}{\kappa}=18.9 \frac{m}{\kappa}=9.45 \frac{g_{t 4}}{\left|\lambda_{4}\right|} .
$$

Using the approximate expression (6), we find

$$
\ln A_{k_{0}} \approx 2.00 \frac{k_{0} \pi}{2 m}=\frac{6.28}{g_{t 4}} .
$$

The general form of eqs. (8), (9) does not guarantee that the prefactor is smaller than the saddle-point action for all values of $g_{t 4}$. Our estimate for the prefactor grows in inverse proportion to the Yukawa coupling and can be significant for small $g_{t 4}$. The calculation of $\lambda_{4}$ is necessary for the determination of the saddle-point action. For the experimental value of the top-quark mass $\left(g_{t 4} \approx 1\right)$ we find $\left|\lambda_{4}\right| \lesssim 0.05$ for all Higgs-field expectation values, in agreement with fig. 1 of ref. 22]. As a result, the prefactor is expected to give only a small correction to the bubble-nucleation rate. We mention at this point that, for the experimental value of the topquark mass $\left(m_{t}=174 \mathrm{GeV}\right)$ 24 and the experimental lower bound on the Higgs-boson mass $\left(m_{H}>90 \mathrm{GeV}\right)$ 25], the difference between the bound from absolute vacuum stability and the more relaxed metastability bound we discussed is small 23. This is an additional reason that makes a more refined determination of the prefactor unnecessary.

The electroweak phase transition: We turn next to the case of radiatively-induced first-order phase transitions. The potential at the scale $k_{0}=T$ has only one minimum, while a new one is generated at a lower scale. Several examples have been given in the past of numerical or analytical solutions of evolution equations, such as eq. (2), that display this behaviour [26, 7]. The basic picture can be obtained by considering the approximate solution of eq. (3) for a theory of two scalar fields (for simplicity we assume the symmetries $\phi_{1} \leftrightarrow-\phi_{1}$, $\left.\phi_{2} \leftrightarrow-\phi_{2}, \phi_{1} \leftrightarrow \phi_{2}\right)$. The expectation value of $\phi_{1}$ is the order parameter for the phase transition, while we consider an interaction $g_{4} \phi_{1}^{2} \phi_{2}^{2} / 4$ between the two fields. The contribution $\propto\left[k_{0}^{2}+\mathcal{M}_{k_{1}}^{2}\right]^{3 / 2}$ in eq. (3) can be expanded in powers of $\mathcal{M}_{k_{1}}^{2} / k_{0}^{2}$. The first term in this expansion, when added to the temperaturedependent part in $U_{k_{0}}$, reproduces the leading hightemperature behaviour of the one-loop perturbative effective potential, as has been checked explicitly in refs. 113, 14, 26. Thus, we can write

$$
U_{k}^{(1)}\left(\phi_{1}\right) \approx-\frac{1}{2} \mu^{2} \phi_{1}^{2}+\frac{1}{4} \lambda \phi_{1}^{4}+\frac{1}{48}(6 \lambda+g) k_{0} \phi_{1}^{2}
$$




$$
-\frac{1}{12 \pi}\left\{\left[k^{2}+\mathcal{M}_{1 k_{1}}^{2}\left(\phi_{1}\right)\right]^{\frac{3}{2}}+\left[k^{2}+\mathcal{M}_{2 k_{1}}^{2}\left(\phi_{1}\right)\right]^{\frac{3}{2}}\right\},
$$

with $\lambda=\lambda_{4} T, g=g_{4} T$. Setting $k=k_{1}=0$ in the above equation results in the standard "mass-gap" equation for the high-temperature potential of this model. As we explained earlier, the calculation of the nucleation rate must be performed for a non-zero value $k_{f}$, with $k_{f}^{2}=\max \left|U_{k_{1}}^{\prime \prime}(1)(\phi)\right|$. For this reason, we use $k=k_{1}=k_{f}$ in the following. For radiative symmetry breaking, $g$ is in general much larger than $\lambda$.

For an approximate estimate of the nucleation rate we can make certain simplifications in eq. (10). We can approximate the mass term of the $\phi_{2}$ field by its zero temperature form: $\mathcal{M}_{2 k}^{2}\left(\phi_{1}\right) \approx g \phi_{1}^{2} / 2$. An improved treatment would take into account the thermal corrections to this mass and the $k$ dependence of the three-dimensional coupling $g$. However, both the above corrections are expected to be small for strongly first-order phase transitions, on which we focus for the rest of our discussion. Moreover, for $g<1$ and $k=k_{1}=k_{f}$, we can neglect $k^{2}$ relative to $\mathcal{M}_{2 k_{1}}^{2}\left(\phi_{1}\right)$. This approximation is not valid only for a small region around the origin, while most of the potential (and especially the barrier) remains unaffected. The term $\propto\left[k^{2}+\mathcal{M}_{1 k_{1}}^{2}\left(\phi_{1}\right)\right]^{3 / 2}$ in eq. (10) can also be neglected, as it is associated with fluctuations of the $\phi_{1}$ field whose self-coupling $\lambda$ is much smaller than $g$. We conclude that, for an estimate of the bubble-nucleation rate for strongly first-order phase transitions, we can use

$$
U_{k_{f}}^{(1)}\left(\phi_{1}\right) \approx \frac{1}{2} m^{2} \phi_{1}^{2}-\frac{1}{3} \gamma \phi_{1}^{3}+\frac{1}{4} \lambda \phi_{1}^{4}
$$

with

$$
m^{2}=\frac{g k_{0}}{24}-\mu^{2}, \quad \gamma=\frac{g^{\frac{3}{2}}}{8 \sqrt{2} \pi}, \quad k_{f}^{2}=\frac{\gamma^{2}}{3 \lambda}-m^{2} .
$$

The potential of eq. (11) is nothing but the high-temperature expansion of the one-loop approximation, for which only the dominant $\phi_{2}$ fluctuations have been considered. The crucial additional piece of information that has been obtained through our discussion is the value of the coarse-graining scale $k_{f}$ at which this potential can be used for the calculation of the bubblenucleation rate.

Through the rescalings $r=\tilde{r} / m, \phi_{1}=\tilde{\phi}_{1} \mathrm{~m}^{2} / \gamma$, the potential can be written as $\tilde{V}\left(\tilde{\phi}_{1}\right)=\tilde{\phi}_{1}^{2} / 2-\tilde{\phi}_{1}^{3} / 3+$ $h \tilde{\phi}_{1}^{4} / 18$, with $h=9 \lambda m^{2} / 2 \gamma^{2}$. For $h \approx 1$ the two minima of the potential have approximately equal depth. The action of the saddle point can be expressed as

$$
S_{k_{f}}=\frac{1}{108 \pi}\left(\frac{g h}{\lambda}\right)^{\frac{3}{2}} \tilde{S}(h),
$$

where $\tilde{S}(h)$ must be determined numerically through $\tilde{V}\left(\tilde{\phi}_{1}\right)$. Similarly, the pre-exponential factor associated

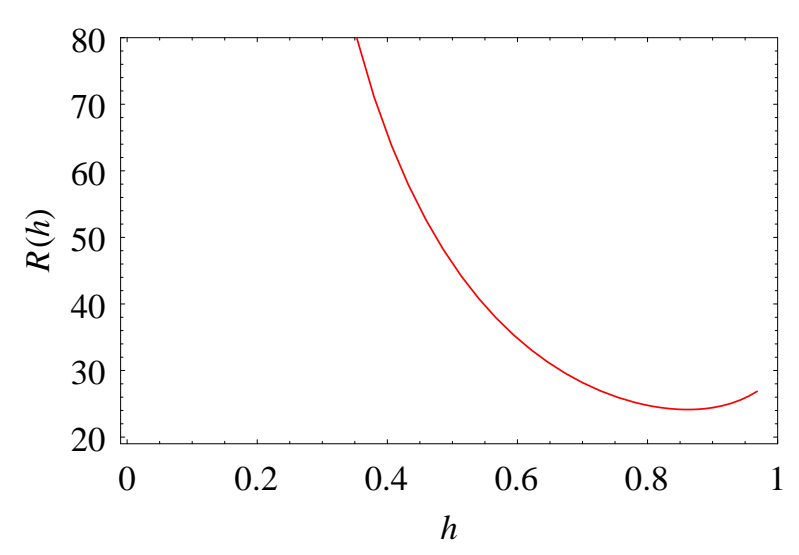

Figure 2: The parameter $R(h)$, defined in eq. (15), as a function of $h$.

with the $\phi_{2}$ field can be estimated from eq. (6) as

$$
\begin{aligned}
\ln A_{2 k_{f}} & \approx-\frac{\pi}{6 \sqrt{2}}\left[\frac{g(3-2 h)}{\lambda}\right]^{\frac{1}{2}} \tilde{A}(h), \\
\tilde{A}^{2}(h) & =\int_{0}^{\infty} \tilde{\phi}_{1}^{2}(\tilde{r}) \tilde{r}^{3} d \tilde{r}
\end{aligned}
$$

with $\tilde{A}(h)$ computed numerically. Finally

$$
\frac{\ln A_{2 k_{f}}}{S_{k_{f}}} \approx-9 \sqrt{2} \pi^{2} \frac{(3-2 h)^{\frac{1}{2}} \tilde{A}(h)}{h^{\frac{3}{2}} \tilde{S}(h)} \frac{\lambda}{g}=-R(h) \frac{\lambda}{g} .
$$

In fig. 2 we plot $R(h)$ as a function of $h$ in the interval $(0,1)$. This function has a minimum $R_{\min } \approx 24$. In the limit $h \rightarrow 0$, our estimate of the prefactor predicts a constant value for $R(h)$. The reason is that the radius $\mathcal{R}$ of the critical bubble becomes very large in this limit, while $\tilde{A}^{2}(h) \propto \mathcal{R}^{4}, \tilde{S}(h) \propto \mathcal{R}^{2}$. However, the approximate expression (6) has not been tested for very large critical bubbles. The divergence of the saddlepoint action and the prefactor in this limit results in low accuracy for our numerical analysis. Typically, our results are reliable for $\tilde{S}(h)$ less than a few thousand. On the other hand, eq. (6) relies on the large- $\ell$ approximation of eq. (5). For increasing $D_{i}$ this approximation breaks down below an increasing value $\ell_{a s}$ and, therefore, eq. (6) is not guaranteed to be valid. However, for the strongly first-order phase transitions that we are discussing, $g$ is larger than $\lambda$ by at least one order of magnitude and values $S_{k_{f}}=\mathcal{O}(100)$ in eq. (13) can be obtained for $h \lesssim 0.9$, where both our numerical and approximate results are reliable.

From eq. (15) and fig. 2 we conclude that

$$
\left|\ln A_{2 k_{f}}\right| / S_{k_{f}} \gtrsim 24 \lambda / g \text {. }
$$

This result is in agreement with fig. 1d, for which $g / \lambda=$ 40 and $S_{k_{f}},\left|\ln A_{2 k_{f}}\right|$ are comparable. Also, the increase of the above ratio for decreasing $g / \lambda$ is in quantitative agreement with the results of ref. 7. More 
specifically, the predicted doubling of $\left|\ln A_{2 k_{f}}\right| / S_{k_{f}}$ for $g / \lambda=20$ is confirmed by fig. 5 of that study. We conclude that the expansion around the saddle point is not convergent, unless $\lambda$ is smaller than $g$ by at least two orders of magnitude. In the limit of zero $\lambda$ (the Coleman-Weinberg limit [19]) our analysis is not sufficient, as corrections $\propto \phi_{1}^{4} \ln \phi_{1}$ that we have neglected become important, and eq. (11) is not a good approximation any more. However, this limit is not relevant for the electroweak phase transition, which is the application we have in mind.

The behaviour of the prefactor associated with the $\phi_{2}$ field can be explained by the form of the differential operators in it. $A_{2 k_{f}}$ involves the ratio

$$
\operatorname{det}\left[-\partial^{2}+\mathcal{M}_{2 k_{f}}^{2}\left(\phi_{b}(r)\right)\right] / \operatorname{det}\left[-\partial^{2}+\mathcal{M}_{2 k_{f}}^{2}(0)\right],
$$

with $\mathcal{M}_{2 k_{f}}^{2}\left(\phi_{1}\right) \approx g \phi_{1}^{2} / 2$. It is easy to check from eq. (10) that, unless $k_{f}^{2} \ll \mathcal{M}_{2 k_{f}}^{2}\left(\phi_{1}\right)$ for all values of $\phi_{1}$ apart from a small region around the origin of the potential, the two-minimum structure cannot be generated. This implies that, in units of $k_{f}$, the function $\mathcal{M}_{2 k_{f}}^{2}\left(\phi_{b}(r)\right)$ takes very large positive values near $r=0$ (see figs 4,5 of ref. [7]). As a result, the lowest eigenvalues of the operator $\operatorname{det}\left[-\partial^{2}+\mathcal{M}_{2 k_{f}}^{2}\left(\phi_{b}(r)\right)\right]$ are much larger than those of $\operatorname{det}\left[-\partial^{2}+\mathcal{M}_{2 k_{f}}^{2}(0)\right]$. This induces a large suppression of the nucleation rate. In physical terms, it implies that the deformations of the critical bubble in the $\phi_{2}$ direction cost excessive amounts of free energy, because the $\phi_{2}$ field is very massive apart from near the origin of the potential. As the $\phi_{2}$ fluctuations are inherent to the system, the total nucleation rate is suppressed considerably when their effect is taken into account.

Based on the above discussion, we can estimate the importance of the pre-exponential factor of the bubblenucleation rate for the electroweak phase transition. We should point out that the rigorous implementation of our approach in gauged Higgs systems must deal with several difficult issues, such as the gauge-invariant definition of the nucleation rate, the gauge-invariant implementation of an ultraviolet cutoff in the prefactor, and the strongly-coupled symmetric phase. However, the basic picture is expected to be very similar to the one in the simple model we considered, at least for strongly first-order phase transitions for which the detailed treatment of the symmetric phase is not crucial. One can use again an effective three-dimensional description, because the longitudinal gauge-field fluctuations develop a large thermal mass and can be integrated out.

The form of the potential is expected to be given by an expression similar to eq. (11). The parameter $\gamma$ includes now contributions $\propto g_{i}^{3}$ from fluctuation determinants (around a constant field configuration) involving fields with couplings $g_{i}$ to the Higgs field. Anal- ogous fluctuation determinants must be included in the prefactor, which becomes a product of terms similar to the one of eq. (14) Each of these terms is $\propto g_{i}$. The final estimate of the magnitude of the prefactor is given by eq. (15), with $\lambda / g$ taking the "effective" value

$$
\left(\frac{\lambda}{g}\right)_{\mathrm{SM}} \approx \frac{\left(4 m_{W}+2 m_{Z}\right) m_{H}^{2}}{4\left(4 m_{W}^{3}+2 m_{Z}^{3}\right)}=0.35\left(\frac{m_{H}}{100 \mathrm{GeV}}\right)^{2} .
$$

For the prefactor $\left|\ln A_{2 k_{f}}\right|$, associated now with the gauge-field sector, to be smaller than $1 / 2$ of the action , the Higgs boson mass must be below $\sim 25 \mathrm{GeV}$. Our conclusions about the large suppression of the nucleation rate by the pre-exponential factor associated with the gauge-field fluctuations is consistent with the results of refs. 化.

Of course, Higgs boson masses below the experimental lower limit of $90 \mathrm{GeV}$ 25] are of academic interest. Moreover, for $m_{H} \gtrsim 80 \mathrm{GeV}$, there is no phase transition in the high-temperature Standard Model [28]. There is the possibility, however, for a sufficiently strongly first-order phase transition for baryogenesis within the Minimal Supersymmetric Standard Model in presence of a very light stop 29]. In this model, the ratio of the "effective" $g$ to $\lambda$ obeys

$$
\begin{aligned}
\left(\frac{\lambda}{g}\right)_{\mathrm{MSSM}} & \gtrsim \frac{\left(4 m_{W}+2 m_{Z}+6 m_{t}\right) m_{H}^{2}}{4\left(4 m_{W}^{3}+2 m_{Z}^{3}+6 m_{t}^{3}\right)} \\
& =0.11\left(\frac{m_{H}}{100 \mathrm{GeV}}\right)^{2} .
\end{aligned}
$$

For $m_{t}=175 \mathrm{GeV}$ 24, in order to have $\left|\ln A_{2 k_{f}}\right|$ smaller than $1 / 2$ of the action, the Higgs boson mass must be below $\sim 45 \mathrm{GeV}$.

It must be pointed out that the additional suppression of the nucleation rate by the prefactor is beneficial for baryogenesis, as it delays the phase transition. This results in larger Higgs expectation values in the interior of the critical bubbles that suppress sphaleron transitions. However, the possible non-convergence of the expansion around the saddle point prohibits any quantitative predictions for the true value of the nucleation rate. Another important point concerns the prefactor associated with the Higgs field fluctuations (the $\phi_{1}$ field

${ }^{4}$ We approximate the fluctuation determinant by a product of determinants, assuming a diagonal mass matrix. Off-diagonal elements, such as the ones appearing in the Goldstone-boson sector in background gauges are $\propto d \phi_{b} / d r$ and, therefore, smaller than the diagonal elements $\propto \phi_{b}^{2}$ for a slowly varying critical-bubble background.

${ }^{5}$ As a very mild criterion of the reliability of the expansion around the saddle point, we demand that the first-order correction (the prefactor) is smaller than $1 / 2$ of the saddle-point action. The bound on the Higgs-boson mass for alternative choices can be obtained easily from eqs. 15 , (16).

6 The fermionic partners of the various fields do not have a zero Matsubara frequency and are not relevant for the effective three-dimensional theory. Moreover, they do not affect the term $\propto \phi_{1}^{3}$ of the potential. 
in our discussion). For weakly first-order phase transitions, such as the ones for Higgs-boson masses larger than the masses of the gauge bosons, this prefactor can enhance the total rate considerably and even compensate the exponential suppression [5, 6]. However, these large corrections signal the breakdown of the saddlepoint approximation.

The presence of significant higher-order corrections in the expansion around the saddle point is confirmed by a large residual dependence of the predicted nucleation rate on the "renormalization" scale $k$. On the contrary, this dependence is very small when the expansion is convergent. This behaviour has been demonstrated numerically in refs. [5]-8] and was not discussed in this paper. Instead we focused here on the presentation of simple analytical expressions (eqs. (3), (5), (6)) that can be used for fast checks of the reliability of the estimated nucleation rates for cosmological phase transitions.

Acknowledgements: We would like to thank R. Barbieri, J. Espinosa and C. Wetterich for helpful discussions. The work of N.T. was supported by the E.C. under TMR contract No. ERBFMRX-CT96-0090.

\section{References}

[1] J. Langer, Ann. Phys. 41, 108 (1967); ibid. 54, 258 (1969); Physica 73, 61 (1974).

[2] S. Coleman, Phys. Rev. D 15, 2929 (1977); C.G. Callan and S. Coleman, Phys. Rev. D 16, 1762 (1977); I. Affleck, Phys. Rev. Lett. 46, 388 (1981); A.D. Linde, Nucl. Phys. B 216, 421 (1983).

[3] E. Weinberg, Phys. Rev. D 47, 4614 (1993); W.N. Cottingham, D. Kalafatis and R. Vinh Mau, Phys. Rev. B 48, 6788 (1993); J. Baacke and V.G. Kiselev, Phys. Rev. D 48, 5648 (1993); M. Gleiser, G.C. Marques and R.O. Ramos, Phys. Rev. D 48, 1571 (1993); G.H. Flores, R.O. Ramos and N.F. Svaiter, Int. J. Mod. Phys. A 14, 3715 (1999).

[4] J. Kripfganz, A. Laser and M.G. Schmidt, Nucl. Phys. B 433, 467 (1995); J. Baacke, Phys. Rev. D 52, 6760 (1995).

[5] A. Strumia and N. Tetradis, Nucl. Phys. B 542, 719 (1999).

[6] A. Strumia, N. Tetradis and C. Wetterich, hepph/9808263, Phys. Lett. B in press.

[7] A. Strumia and N. Tetradis, Nucl. Phys. B 554, 697 (1999).

[8] A. Strumia and N. Tetradis, hep-ph/9904246, Nucl. Phys. B in press.

[9] J. Berges, N. Tetradis and C. Wetterich, Phys. Lett. B 393, 387 (1997); J. Berges and C. Wetterich, Nucl. Phys. B 487, 675 (1997).

[10] C. Wetterich, Nucl. Phys. B 352, 529 (1991); Z. Phys. C 57, 451 (1993); it ibid. 60, 461 (1993).
[11] C. Wetterich, Phys. Lett. B 301, 90 (1993).

[12] N. Tetradis and C. Wetterich, Nucl. Phys. B 422, 541 (1994); T.R. Morris, Phys. Lett. B 329, 241 (1994).

[13] N. Tetradis and C. Wetterich, Nucl. Phys. B 398, 659 (1993); Int. J. Mod. Phys. A 9, 4029 (1994).

[14] N. Tetradis, Nucl. Phys. B 488, 92 (1997).

[15] A. Ringwald and C. Wetterich, Nucl. Phys. B 334, 506 (1990);

[16] N. Tetradis and C. Wetterich, Nucl. Phys. B 383, 197 (1992).

[17] C. Wetterich, Mod. Phys. Lett. A 11, 2573 (1996).

[18] W. Buchmüller, Z. Fodor, T. Helbig and D. Walliser, Ann. Phys. 234, 260 (1994); A. Kusenko, Phys. Lett. B 358, 47 (1995); A. Kusenko, K. Lee and E. Weinberg, Phys. Rev. D 55, 4903 (1997).

[19] S. Coleman and E. Weinberg, Phys. Rev. D 7, 1888 (1973).

[20] N. Cabibbo, L. Maiani, G. Parisi and R. Petronzio, Nucl. Phys. B 158, 295 (1979); M. Lindner, Z. Phys. C 31, 295 (1986); M. Sher, Phys. Rep. 179, 273 (1989); M. Lindner, M. Sher and H. Zaglauer, Phys. Lett. B 228, 139 (1989); M. Sher, Phys. Lett. B 317, 159 (1993); G. Altarelli and I. Isidori, Phys. Lett. B 337, 141 (1994); J.A. Casas, J.R. Espinosa and M. Quirós, Phys. Lett. B 342, 171 (1995).

[21] G. Anderson, Phys. Lett. B 243, 265 (1990).

[22] P. Arnold and S. Vokos, Phys. Rev. D 44, 3620 (1991).

[23] J.R. Espinosa and M. Quirós, Phys. Lett. B 353, 257 (1995).

[24] F. Abe et al. (CDF Collaboration), Phys. Rev. Lett. 82, 271 (1999); B. Abbott et al. (D0 Collaboration), Phys. Rev. D 60, 052001 (1999).

[25] Review of Particle Properties, Particle Data Group, C. Caso et al., Eur. Phys. J. C 3, 1 (1998).

[26] S. Bornholdt, N. Tetradis and C. Wetterich, Phys. Lett. B 348, 89 (1995); Phys. Rev. D 53, 4552 (1996); S. Bornholdt, P. Büttner, N. Tetradis and C. Wetterich, Int. J. Mod. Phys. A 14, 899 (1999); D. Litim, C. Wetterich and N. Tetradis, Mod. Phys. Lett. A 12, 2287 (1997); N. Tetradis, Phys. Lett. B 431, 380 (1998); B. Bergerhoff, D. Litim, S. Lola and C. Wetterich, Int. J. Mod. Phys. A 11, 4273 (1996); B. Bergerhoff, F. Freire, D. Litim, S. Lola and C. Wetterich, Phys. Rev. B 53, 5734 (1996).

[27] N. Tetradis and D. Litim, Nucl. Phys. B 464, 492 (1996).

[28] M. Reuter and C. Wetterich, Nucl. Phys. B 408, 91 (1993); B. Bergerhoff and C. Wetterich, Nucl. Phys. B 440, 171 (1995); preprint HD-THEP-95-37, hepph/9508352; W. Buchmüller and O. Philipsen, Phys. Lett. B 354, 403 (1995); Nucl. Phys. B 443, 47 (1995); K. Kajantie, M. Laine, K. Rummukainen and M. Shaposhnikov, Phys. Rev. Lett. 77, 2887 (1996).

[29] M. Carena, M. Quiros and C. Wagner, Phys. Lett. B 380, 81 (1996); M. Carena, M. Quiros, A. Riotto, I. Vilja and C. Wagner, Nucl. Phys. B 503, 387 (1997). 